\title{
JETS, DISK WINDS, AND WARM DISK CORONAE IN CLASSICAL T TAURI STARS
}

\author{
JOHN KWAN \\ Department of Physics and Astronomy, \\ University of Massachusetts, \\ Amherst, MA 01003, USA
}

\begin{abstract}
Arguments, based on analysis of the forbidden line emission, are summarized that point to two components of mass ejection in classical $\mathrm{T}$ Tauri stars. A low-speed wind originates from the accretion disk at between $\sim 0.05 \mathrm{AU}$ and $z 3 \mathrm{AU}$ from the star, and a high-speed flow, which becomes collimated into a jet within $\lesssim 100 \mathrm{AU}$, originates as a wind from either the star or the very inner part of the accretion disk. The [OI] $\lambda 5577$ emission in the low-speed component also requires the presence of a warm disk corona, with an electron density of $\sim 10^{7} \mathrm{~cm}^{-3}$ and a temperature of $\sim 8000 \mathrm{~K}$. Additional indication of a warm disk corona comes from analysis of the central absorptions seen in the profiles of the hydrogen Balmer lines. The need to heat the disk corona implies that a substantial fraction of the energy released in the accretion of matter through the disk may be dissipated at the disk surface.
\end{abstract}

\section{Introduction}

In this symposium there are many theoretical discussions on how winds and jets are launched from a classical T Tauri star (cTTS) or its accretion disk (see the papers in this volume by Camenzind; Heyvaerts \& Norman; Pudritz \& Ouyed; Shu \& Shang; and the poster papers by Ferreira, Pelletier, \& Appl (1997); and Paatz \& Appl 1997). Here the question is posed from the observational point of view: what do the observations tell about the location and properties of the mass ejection? In particular, arguments, based on analysis of the forbidden lines, are presented that indicate there are two components of mass ejection: a high-speed component, originating from the star or the very inner part of the accretion disk, that becomes collimated 
into a jet, and a comparatively much slower wind originating from the outer part of the disk up to a distance of several AUs from the star.

The [OI] $\lambda 5577$ emission in the low-speed component also points to the presence of a disk corona with a temperature of $\sim 8000 \mathrm{~K}$ and an electron density of $\sim 10^{7} \mathrm{~cm}^{-3}$. It is this warm corona that extends vertically from the disk into the slow disk wind. Additional indication of a warm disk corona may come from the observed profiles of the hydrogen Balmer lines. It is suggested that the central absorptions seen in those lines are produced by a disk corona within a distance of $\lesssim 10^{13} \mathrm{~cm}$ from the star.

Throughout this paper I shall constantly refer to the fine sets of [OI] and hydrogen line profiles that Hartigan, Edwards, \& Ghandour (1995, hereafter HEG) and Edwards et al. (1994, hereafter EHGA) carefully extract from their echelle spectra. In the next section I shall describe how the observed forbidden line profiles are most readily explained by two separate outflow components. In $\S 3$ the arguments for a warm disk corona, based on the [OI] $\lambda 5577 /[\mathrm{OI}] \lambda 6300$ intensity ratio and the Balmer line central absorption, are put forward. The implications of a warm disk corona and a disk wind on the process of mass accretion through the disk is discussed in $\S 4$. Many of the thoughts, arguments, and calculations were originally presented in Kwan \& Tademaru (1988; 1995, hereafter KT) and Kwan (1997).

\section{Two Components of Mass Ejection: a Jet and a Disk Wind}

When the OI forbidden lines were first examined, it was realized right away that the generally stronger emission blueward of the stellar velocity indicates the presence of an obscuring disk and that the double-peaked shape of the line profile seen in a fraction of the stars cannot be produced by a geometric structure with spherical symmetry (Appenzeller, Jankovics, \& Östreicher 1984; Edwards et al. 1987). The recent high-resolution line profiles of 32 cTTS obtained by HEG provide a large sample for analysis. Referring to their Figure 5, which shows the [OI] $\lambda 6300$ profiles, it is seen that a peak located, in the rest frame of the star, at zero velocity or slightly to the blue (by $\sim 5 \mathrm{~km} \mathrm{~s}^{-1}$ ) is always present. This is often referred to as the low-velocity peak. In about a third of the stars another peak, displaced blueward by $\chi 100 \mathrm{~km} \mathrm{~s}^{-1}$, is prominent. This blue or high-velocity peak is stronger than the low-velocity peak in two stars and comparable to or weaker than the low-velocity peak in the others. In three stars (AS 353A, RW Aur, DF Tau) a third peak, displaced to the red of zero velocity by an amount comparable to the shift of the blue peak, also stands out.

The more frequent appearance of the blue peak than the red peak, and, when the blue peak is not conspicuous, the generally stronger emission blueward of zero velocity strongly suggest the presence of a disk that in 
most cases obscures the receding part of an outflowing wind. It follows that the red peak is most likely the counterpart of the blue peak. Considering only the gas flow in front of the disk then, the prevalence of the low-velocity peak and the occasional double-peaked line shape need to be explained. The models proposed can be divided according to the two premises: a onecomponent model in which a high-speed non-isotropic wind produces both the low-velocity and blue peaks as a result of different projections of the velocity vectors along the line of sight (Edwards et al. 1987; Hartmann \& Raymond 1989; Safier 1993; Ouyed \& Pudritz 1994), and a two-component model in which a high-speed flow produces the blue peak and a much slower wind produces the low-velocity emission.

There are several lines of argument, supported by observational results, that indicate the one-component model is unlikely. In such a model there is a strong relation between the emission properties of the blue and lowvelocity peaks, since the blue peak can appear, from a different viewing angle, as a low-velocity peak and vice versa. Given that the viewing angle is arbitrary, the model will be hard pressed to explain the much more frequent appearance of the low-velocity peak than the blue peak. It will also stipulate similar physical conditions for the blue and low-velocity emissions. Observed profiles of other forbidden lines (Hamann 1994; Figs 6, 7, \& 8 of HEG), on the other hand, show that, in comparison with the [OI] $\lambda 6300$ line, the [OI] $\lambda 5577$ line has no or only a weak blue peak, while the [SII] $\lambda 6731$ and [NII] $\lambda 6583$ lines in quite a few stars have a prominent blue peak but no low-velocity peak. Comparisons of the observed intensity ratios with excitation calculations reveal that the electron densities responsible for the [OI] $\lambda 5577$ low-velocity emission and the [SII] $\lambda 6731$ blue emission are $\gtrsim 5 \times 10^{6} \mathrm{~cm}^{-3}$, and $\lesssim 3 \times 10^{4} \mathrm{~cm}^{-3}$ respectively (Hamann 1994; HEG; $\mathrm{KT})$. This large systematic difference in the electron density is not easily effected in a one-component model. The strong contrast of the [SII] $\lambda 6731$ and [NII] $\lambda 6583$ profiles versus the [OI] $\lambda 5577$ profile can actually be used, in those stars in which the two peaks appear blended together in the [OI] $\lambda 6300$ profile, to separate out the two contributions (see Fig. 8 of HEG). A third argument against the one-component model comes from high resolution long-slit spectral imaging of several cTTS (Solf \& Böhm 1993; Hirth, Mundt, \& Solf 1994; see also the paper by Solf in this volume). The emission at the blue peak is found to be more spatially extended along an axis than the low-velocity emission. This systematic dependence of spatial distribution on velocity is not easily reconciled in a one-component model that produces a low observed velocity via geometric projection.

The constraints inherent in a one-component model are lifted in a model with two emission components. The blue and low-velocity peaks can be examined and modelled separately. In this light the gas kinematics needed to 
produce the two peaks are found to be very different. Assuming a geometry with axial symmetry, and noting that the forbidden lines are optically thin, an observed line profile can be thought of as the sum of the emission profiles from many axisymmetric rings of particles. Let the velocity components of a particle in the rest frame of the star be denoted by $v_{r}, v_{\phi}$, and $v_{z}$ in a cylindrical co-ordinate system with the symmetry axis along $z$. The basic line profile from an axisymmetric ring of particles is symmetric about the observed velocity $v_{l}=-v_{z} \cos i$. It extends from this midpoint to a width on each side of $\left(v^{2}-v_{z}^{2}\right)^{0.5} \sin i$, where $i$ is the inclination of the line of sight from the $z$-axis, and $v$ is the velocity magnitude. The amplitude of the line is lowest at the midpoint and highest at the two edges, so the basic profile has a double-peaked appearance.

In several cTTS the blue peak is prominently displaced (by $\geq 80 \mathrm{~km} \mathrm{~s}^{-1}$ ) from zero velocity and is fairly sharp (see the [OI] $\lambda 6300$ line in AS 353A, DO Tau, and CW Tau, and the [SII] $\lambda 6731$ line in DO Tau, DD Tau, DL Tau, and DK Tau from Figs. 5 \& 7 of HEG), with the half-width at halfmaximum being less than $\sim 1 / 4$ of the displacement of the peak. To produce it from a superposition of many basic profiles, a substantial contribution is needed from axisymmetric rings of particles with $v_{z}$ large and within several percent of $v$. This condition indicates a high-speed flow that becomes collimated into a jet. The result from long-slit spectral imaging that the emission at the blue peak is spatially elongated along the direction marked by an extended optical jet and/or Herbig-Haro objects (Solf \& Böhm 1993; Hirth, Mundt, \& Solf 1994) provides further corroboration.

The fraction of cTTS in HEG's sample showing a blue peak is $\sim 1 / 2$ if one includes the stars DD Tau, HN Tau, AA Tau, and Lk Ca 8 in which the blue and low-velocity peaks (while blended together in the [OI] $\lambda 6300$ profile) can be separated out by utilizing the [SII] $\lambda 6731$, [NII] $\lambda 6583$, and [OI] $\lambda 5577$ profiles. Then, given the possibility that physical conditions amenable to producing strong forbidden line emission may not always be present, particularly if excitation of the gas in the jet is via internal shocks produced by a time-dependent flow, the above fraction suggests that a highspeed flow that becomes collimated within $\lesssim 100 \mathrm{AU}$ of the star may be a common characteristic of cTTS.

Examining the low-velocity peak in the [OI] $\lambda 6300$ profile (see Fig. 5 of HEG), it is seen that the peak is blue-shifted from the stellar velocity by $\lesssim 5 \mathrm{~km} \mathrm{~s}^{-1}$ and the line intensity falls off roughly symmetrically and fairly sharply from the peak to values of $\sim \pm 100 \mathrm{~km} \mathrm{~s}^{-1}$. The fact that the low-velocity peak is always present and blue-shifted, but only slightly, indicates that, whatever the viewing angle, the particles producing the lowvelocity peak has a positive but small $v_{z}\left(\$ 10 \mathrm{~km} \mathrm{~s}^{-1}\right)$. To produce the line shape from a superposition of many basic profiles, it is also necessary 
that the particles have a broad range in $v$ from a value $\$ 10 \mathrm{~km} \mathrm{~s}^{-1}$ (the instrumental resolution of the spectra) to $\sim 100 \mathrm{~km} \mathrm{~s}^{-1}$. A casual comparison of observed profiles with theoretical ones calculated assuming that the number of particles per unit velocity interval in the upper state of a forbidden line is proportional to $v^{\alpha}$ finds that the index $\alpha$ lies between 0 and -1 , indicating that a substantial fraction of the emitting particles have low speeds. Furthermore, it is difficult to avoid a dip in the intensity right at line center unless there are particles with speeds about $10 \mathrm{~km} \mathrm{~s}^{-1}$ or lower (see Fig. 2 of KT).

The broad distribution in $v$ (from $\lesssim 10$ to $\sim 100 \mathrm{~km} \mathrm{~s}^{-1}$ ) needed to produce the line shape of the low-velocity peak suggests that Keplerian rotation from $\sim 0.05 \mathrm{AU}$ out to $z 3 \mathrm{AU}$ is a likely cause. The small but positive $v_{z}$ of the gas then signals a wind emanating from the disk. This hypothesis is consistent with the observational result that, when the lowvelocity peak is clearly distinct from the blue peak in the [OI] $\lambda 6300$ profile and [SII] $\lambda 6731$ is also observed to have low-velocity emission (in DG Tau, CW Tau, DQ Tau, UY Aur), the progression of the blue-shifts among the [OI] $\lambda 5577,[\mathrm{OI}] \lambda 6300$, and [SII] $\lambda 6731$ lines, which have successively lower critical electron densities for collisional de-excitation, indicates a higher $v_{z}$ at a lower electron density (Solf \& Böhm 1993; Hirth, Mundt, \& Solf 1994; HEG).

In CW Tau and DG Tau the [OI] $\lambda 6300$ luminosity in the low-velocity component is high, $\sim 4 \times 10^{-3} L_{\odot}$ (including a factor of two to account for obscuration by the disk), and it places a constraint on the size of the emission region. Assuming an [OI] $\lambda 6300$ excitation temperature given by $T_{\lambda 6300}$ in the range between $6 \times 10^{3}$ and $10^{4} \mathrm{~K}$, oxygen to be all neutral, and a solar composition, the mass needed to produce the above luminosity is $M \simeq$ $5 \times 10^{-8}\left(8 \times 10^{3} \mathrm{~K} / T_{\lambda 6300}\right)^{3} M_{\odot}$. For a mass loss rate given by $\dot{M}$, an outflow speed given by $v_{t}$, the linear size along the outflow direction is then $l \sim$ $M v_{t} / \dot{M} \sim 1.6 \times 10^{13}\left(8 \times 10^{3} \mathrm{~K} / T_{\lambda 6300}\right)^{3}\left(v_{t} / 10 \mathrm{~km} \mathrm{~s}^{-1}\right)\left(10^{-7} M_{\odot} y r^{-1} / \dot{M}\right)$ $\mathrm{cm}$. This result indicates that, at least for these two stars, the low-velocity emission cannot originate from the neighborhood of the star. It adds credence to the hypothesis of an extended disk wind.

The above calculation, in the disk wind hypothesis, determines the vertical height of the [OI] $\lambda 6300$ emission from the disk if $v_{t}$ is replaced by $v_{z}$. A similar manipulation using the [SII] $\lambda 6731$ luminosity in CW Tau and DG Tau, which is $\sim 7 \times 10^{-4}$ and $\sim 2 \times 10^{-3} L_{\odot}$ (HEG), obtains, assuming sulphur is all SII, the corresponding vertical height of $\sim 25\left(8 \times 10^{3} \mathrm{~K} / T_{\lambda 6731}\right)^{2.6}\left(v_{z} / 10 \mathrm{~km} \mathrm{~s}^{-1}\right)\left(10^{-7} M_{\odot} y r^{-1} / \dot{M}\right)$ AU and $\sim 70$ AU respectively, where $T_{\lambda 6731}$ is the [SII] $\lambda 6731$ excitation temperature. Given the large uncertainties in $T_{\lambda 6731}, v_{z}$, and $\dot{M}$, these heights are roughly compatible with the observed values of $\sim 150$ and $\sim 50 \mathrm{AU}$ for the [SII] $\lambda 6731$ 
emission in CW Tau and DG Tau respectively (Hirth, Mundt, \& Solf 1994; Solf \&.Böhm 1993).

If the origin of the low-velocity emission is a disk wind, that of the highvelocity emission is less clear. Observed Balmer lines of hydrogen sometimes show blue absorptions at $\varsigma-100 \mathrm{~km} \mathrm{~s}^{-1}$ (EHGA). The need to maintain population in the $n=2$ excited level of hydrogen in order to produce the absorption indicates that the high-speed outflowing gas responsible probably lies close to the star. Theoretically it also makes sense that the gas attaining a terminal speed of $\sim 300 \mathrm{~km} \mathrm{~s}^{-1}$ starts originally at a gravitational potential from where the escape velocity is not much smaller, otherwise the stresses built up to produce the acceleration will be unnecessarily excessive. In the case of the disk wind producing the low-velocity emission, for example, $v_{z} \cos i$, as revealed by the spectral imaging studies (Hirth, Mundt, \& Solf 1994; Solf \& Böhm 1993), reaches a terminal value of only 12 and $\sim 43 \mathrm{~km} \mathrm{~s}^{-1}$ in CW Tau and DG Tau respectively. It is likely, therefore, that the high-speed gas that ultimately becomes collimated originates as a wind from either the star or the very inner part of the accretion disk.

\section{A Warm Disk Corona}

Besides conveying information on the kinematics of the gas through their profiles, the forbidden lines also reveal through their intensities the physical conditions giving rise to their excitation. The intensity ratio [OI] $\lambda 5577 /[\mathrm{OI}]$ $\lambda 6300$, in particular, strongly constrains the electron density $n_{e}$ and temperature $T$. It will be shown that its value in the low-velocity component suggests the presence of a warm disk corona $\left(n_{e} \sim 10^{7} \mathrm{~cm}^{-3}, T \sim 8000 \mathrm{~K}\right)$ out to a distance of $\chi^{3} \mathrm{AU}$ from the star. In addition, analysis of the central absorptions seen in the Balmer lines of hydrogen points to the absorption gas being within $10^{13} \mathrm{~cm}$ from the star and at a height above the disk that is large in relation to its distance (i.e., $z / r \not 0.3$ ). The presence of high density gas $\left(\sim 10^{10} \mathrm{~cm}^{-3}\right)$ at that location is also indicative of a warm disk corona. These two lines of evidence are presented sequentially below.

\section{1. [OI] $\lambda \lambda 6300,5577$ EMISSION}

The [OI] $\lambda 5577$ line originates from an excited level that is $\sim 2.2 \mathrm{eV}$ above the upper level of [OI] $\lambda 6300$. It has a spontaneous emission rate of $\sim 1.3$ $\mathrm{s}^{-1}$ versus that of $\sim 5 \times 10^{-3} \mathrm{~s}^{-1}$ for [OI] $\lambda 6300$. The conditions favoring $\lambda 5577$ over $\lambda 6300$ emission are then a high electron density and a high temperature. Observationally, in the low-velocity component [OI] $\lambda 5577 /[\mathrm{OI}]$ $\lambda 6300$ lies between 0.1 and 1 (HEG). To produce a ratio of 0.2 , for example, the electron density needed is $\sim 5 \times 10^{6} \mathrm{~cm}^{-3}$ at $T=10^{4} \mathrm{~K}$, and increases 
to $\sim 4 \times 10^{7} \mathrm{~cm}^{-3}$ at $T=6000 \mathrm{~K}$ (see Fig. 3 of KT). For ease of reference, $T=8000 \mathrm{~K}$ and $n_{e}=10^{7} \mathrm{~cm}^{-3}$ shall be taken as representative values.

In the disk wind hypothesis the low-velocity emission originates at a distance out to $\gtrsim 3 \mathrm{AU}$ from the star. To produce an $n_{e}$ of $10^{7} \mathrm{~cm}^{-3}$ that far from the star places a taxing demand on the ionization source. It is readily shown that photoionization by the stellar or veiling continuum is inadequate. If $r \sim 2.5 \times 10^{13} \mathrm{~cm}$ denotes roughly half the extent of the lowvelocity emission along the disk, the number of ionizing photons incident per second per unit area at $r$ needs to be $\sim n_{e}^{2} \alpha_{r} r$, where $\alpha_{r} \simeq 2.6 \times$ $10^{-13} \mathrm{~cm}^{3} \mathrm{~s}^{-1}$ is the radiative recombination coefficient. The corresponding luminosity, assuming the photons to have energy $h \nu$, is then $\sim n_{e}^{2} \alpha_{r} 4 \pi r^{3} h \nu$ $\sim 2.8 \times 10^{-2}\left(n_{e} / 10^{7} \mathrm{~cm}^{-3}\right)^{2}\left(r / 2.5 \times 10^{13} \mathrm{~cm}\right)^{3}(h \nu / 13.6 \mathrm{eV}) L_{\odot}$. It is stronger than the Lyman continuum of the veiling flux, if the latter is represented by a blackbody of luminosity $\leq 1 L_{\odot}$ and temperature $\leq 2 \times 10^{4}$ $\mathrm{K}$. The Lyman continuum will also suffer severe intervening attenuation by the gas infalling onto the star along stellar magnetic field lines. The required ionizing luminosity also greatly exceeds the observed X-ray luminosity of $\sim 3 \times 10^{-5} L_{\odot}$ from cTTS (Neuhäuser et al. 1995).

Assuming that the energy source is heat, hydrogen excitation calculations have been carried out that include collisional processes involving the ground and $n=2$ levels, Balmer photoionization, two-photon radiative decay, and electron-proton recombinations. The proton density is sufficiently high to ensure that the $2 \mathrm{~s}$ and $2 \mathrm{p}$ level populations are in the thermalequilibrium ratio of $1: 3$, and the decay route through escape of Ly $\alpha$ photons has been neglected in order to obtain a conservative estimate of the physical conditions. To produce an $n_{e}$ of $10^{7} \mathrm{~cm}^{-3}$ at a distance of $r=2.6 \times 10^{13}$ $\mathrm{cm}$, for example, it is found that a temperature of greater than $10^{4}$, equal to 9000 , and slightly less than $8000 \mathrm{~K}$ is needed if the hydrogen density $n_{H}$ equals $10^{8}, 10^{9}$, and $10^{10} \mathrm{~cm}^{-3}$ respectively (see Fig. 4 of Kwan 1997).

The local heating rate needed to excite hydrogen and other elements, such as OI and FeII, that produce strong cooling, is high. In the above example of $n_{H}=10^{9} \mathrm{~cm}^{-3}, r=2.6 \times 10^{13} \mathrm{~cm}$, and $T=9000 \mathrm{~K}$, it is $\sim 1.5 \times 10^{-8} \mathrm{erg} \mathrm{s}^{-1} \mathrm{~cm}^{-3}$. This heating rate has to be maintained over a $z$ height that is at least as large as the product of the sound speed and the electron-proton recombination time, which is $\sim 3.6 \times 10^{11} \mathrm{~cm}$. The required energy input rate per unit area on the disk at $r=2.6 \times 10^{13} \mathrm{~cm}$ is then $\sim 1.1 \times 10^{4} \mathrm{erg} \mathrm{s}^{-1} \mathrm{~cm}^{-2}$. Presumably the energy is ultimately derived from accretion. Assuming a stellar mass of $0.7 M_{\odot}$, the corresponding mass accretion rate needed is $\sim 1.3 \times 10^{-7} M_{\odot} y r^{-1}$. 


\subsection{BALMER LINE CENTRAL ABSORPTION}

In 5 of the 15 cTTS that EHGA obtained profiles of the hydrogen Balmer lines a strong absorption at line center is prominent, even in $\mathrm{H} \delta$. This absorption must occur within $3 \times 10^{14} \mathrm{~cm}$ from the star, otherwise, if the $\mathrm{n}=2$ level is populated by electron-proton recombinations, the $\mathrm{H} \alpha$ luminosity from the absorption region will itself exceed the observed value, or, if the $n=2$ level is populated by collisional excitation, the heating luminosity needed will be prohibitively high (Kwan 1997). This constraint, together with the fairly common occurrence of the central absorption, argues strongly for an origin that is intrinsic to the star and its accretion disk.

The deep central absorption in $\mathrm{H} \beta, \mathrm{H} \gamma$, or $\mathrm{H} \delta$ extends far below the continuum in AA Tau, DR Tau, and RW Aur (see Fig. 3 of EHGA). The veiling continua in these three stars are, respectively, about $0.2,10$, and 3.4 times the stellar photospheric continua (EHGA). In AA Tau then the absorption gas must be able to cover, along the line of sight, a large fraction of the stellar surface, while in DR Tau and RW Aur, assuming that the veiling continuum is produced near the polar region of the star, some absorption gas must lie at least one stellar radius above the disk plane.

There are two possible explanations for the incidence of 5 out of 15 stars showing a central absorption. One is that the 5 stars are the only ones in the sample with an absorption region, which covers the entire $4 \pi$ solid angle about the star. The other is that the absorption region is present in each of the 15 stars but it covers, on average, only a fraction $f \sim 5 / 15$ of the $4 \pi$ solid angle. The first explanation is less likely because of the following argument. It is reasonable to assume that all the Lyman transitions are ineffective decay routes owing to their very large opacities. Absorption of an $\mathrm{H} \alpha$ photon then leads to emission of another $\mathrm{H} \alpha$ photon. If the absorption gas covers the whole $4 \pi$ solid angle, there will be no net loss of $\mathrm{H} \alpha$ photons in traversing the absorption region. The $\mathrm{H} \alpha$ photons can be re-distributed in frequency (as in the standard case of a P-Cygni profile being produced by a spherical-symmetric outflow), but here the absorption is centered at the stellar velocity in three stars and only slightly shifted to the blue in the other two, so the scattering will not produce a broad frequency re-distribution. There can be a large bulk motion transverse to the line of sight, but in this case the motion, most probably rotation, will itself point to a geometric structure that only partially covers the $4 \pi$ solid angle. Furthermore, following absorptions of $\mathrm{H} \beta, \mathrm{H} \gamma, \mathrm{H} \delta$, etc. within the region, there is net $\mathrm{H} \alpha$ emission. Thus it is difficult to comprehend the strong $\mathrm{H} \alpha$ absorptions seen in AA Tau, GK Tau, and RW Aur if the absorption gas covers the star completely. It seems more likely that the absorption gas is 
present in each star, but the covering fraction probably varies substantially from star to star.

In DK Tau and GK Tau the central absorption in $\mathrm{H} \beta$ extends below the continuum, but that in $\mathrm{H} \gamma$ and $\mathrm{H} \delta$ are successively weaker (EHGA). These results, together with the fact that the $\mathrm{H} \beta, \mathrm{H} \gamma$, and $\mathrm{H} \delta$ opacities decrease in order, suggest that the line profile, in the absence of the absorption, is centrally peaked. The absorption gas will then need to lie at a distance from the star that is beyond the emission region of the Balmer lines.

Of the five stars with a Balmer line central absorption four, among a group of eight in the sample, also show a red absorption in one or more Balmer lines. The remaining, DR Tau, has a red absorption in its $\mathrm{Na} D$ line (EHGA). The red absorption is interpreted as being produced by infalling gas along stellar magnetic field lines that intercept the disk (Hartmann, Hewett, \& Calvet 1994; EHGA). It appears that when a central absorption is seen, a red absorption is also present.

Considering all the arguments and interpretations presented above, it is believed that the gas producing the central absorption is located beyond the magnetospheric accretion column in order to be able to cover the stellar surface, the veiling continuum, and the region producing the hydrogen emission lines. The moderate covering fraction subtended by this absorption region, the small radial motion of the gas, and the possible correlation of the central absorption with the red absorption indicate that the gas is confined more to the equatorial than to the polar region. The need for some of the absorption gas to lie one stellar radius or more above the disk in order to cover the veiling continuum source suggests that a disk corona is present.

To determine the physical conditions needed to produce the Balmer line central absorption, calculations of the $n=2$ level population have been carried out. The Lyman continuum is assumed to be completely absorbed by the gas in the magnetospheric accretion column. X-ray photoionization is also judged to be ineffective (Kwan 1997). In addition to thermal collisions and Balmer photoionization, two processes that utilize the continuum between 12 and $13.6 \mathrm{eV}$ are included (Kwan \& Alonso-Costa 1988). The first is ionization from the $2 \mathrm{p}^{3}{ }^{2} \mathrm{D}$ state of NI. That state, lying at $\sim 2.4 \mathrm{eV}$ above the ground state, can be ionized by photons more energetic than $12.16 \mathrm{eV}$. Charge-exchange of NII with hydrogen will then produce hydrogen ionization. The second process is absorption of $\operatorname{Ly} \beta$ in the damping wings, followed by a spontaneous decay to the $2 \mathrm{~s}$ level. These two processes reduce the heat input needed to produce the hydrogen excitation if the veiling continuum at those photon energies is not too weak.

The details of the calculations are presented in Kwan(1997). Here the main results are summarized. Assuming a continuum luminosity of 0.3 
$L_{\odot}$ between 3.4 and $13.6 \mathrm{eV}$, and an energy distribution $\left(\mathrm{erg} \mathrm{s}^{-1} \mathrm{~Hz}^{-1}\right)$ that is proportional to $\nu^{-3}$, it is found that, over the distance range $3.2 \times$ $10^{11} \mathrm{~cm} \leq r \leq 10^{13} \mathrm{~cm}$, the temperature needed to produce an $\mathrm{H} \delta$ opacity near unity is $\sim 3300, \sim 4500$, and $z 7000 \mathrm{~K}$ if the hydrogen density $n_{H}$ equals $10^{11}, 10^{10}$, and $10^{9} \mathrm{~cm}^{-3}$ respectively. For the same luminosity of $0.3 L_{\odot}$ but a $\nu^{-5}$ frequency dependence, the required temperature is about 4000 and $5500 \mathrm{~K}$ for $n_{H}=10^{11}$ and $10^{10} \mathrm{~cm}^{-3}$, respectively, while the corresponding temperature required in the absence of NI excited-state ionization is about 5600 and $6500 \mathrm{~K}$.

The above conditions of a fairly high density and temperature, together with the need for the gas to be present at a substantial height above the disk (i.e., $z / r z 0.3$ ) in order to account for the incidence of absorption, corroborates the argument for a warm disk corona.

\section{Discussion}

The disk-wind origin for the low-velocity forbidden line emission and the presence of a warm disk corona have important implications on the accretion process through the disk, so it is important to establish their validity first. The determination of $n_{e} \sim 10^{7} \mathrm{~cm}^{-3}$ and $T \sim 8000 \mathrm{~K}$ needed to produce the low-velocity [OI] $\lambda 5577$ emission is firm. It is the location of this emission that has to be ascertained. Spatial imaging with the Hubble Space Telescope may reveal the extent of the emission along the disk. Observations with a very high spectral resolution to compare the [OI] $\lambda 5577$ and [OI] $\lambda 6300$ profiles near the low-velocity peak will provide strong constraints on the detailed modelling.

On the interpretation of the Balmer line central absorption as being caused by a warm disk corona, several self-consistency checks can be made. Even though a strong continuum between 12 and $13.6 \mathrm{eV}$ is not essential, it facilitates the hydrogen excitation with a minimum need for heat input. It is useful to measure the continuum shape beyond the Balmer edge, not only to make the excitation calculations more definite, but also to determine the veiling continuum luminosity more accurately. Another check lies in the shape of the Paschen lines. These lines are optically thin in the absorption region, so they should not possess central absorptions. Recent observations of $\mathrm{Pa} \beta$ in $\mathrm{T}$ Tauri stars show that indeed they do not (Folha, private communication; see also the paper by Folha, Emerson, \& Calvet in this symposium). A third check is the correlation between the central and red absorptions. A large sample of cTTS to examine the central, red, and blue absorptions will not only elucidate the geometrical distributions of the different gas flows close to the star, but may even help determine whether the high-speed outflow, which becomes collimated into a jet, originates from 
the star or the very inner part of the accretion disk.

If the postulate of a warm disk corona is correct, then the energy input rate needed to maintain it is considerable. As estimated in $\S 3.1$, it entails a mass accretion rate of $\sim 1.3 \times 10^{-7} M_{\odot} y r^{-1}$. While this estimate is quite uncertain, it nonetheless points to a substantial fraction of the energy released from the accretion of matter through the disk being dissipated at the disk surface. This is contrary to the usual assumption of dissipation of all the energy in the form of blackbody radiation from the bulk of the disk. One way for this to occur, in light of the recent emphasis by Balbus \& Hawley (1991) of a magnetohydrodynamic instability, may be the damping at the disk surface of Alfven waves that are generated as a result of the instability.

Even if most of the energy dissipation occurs at the disk surface, half of the radiation emitted in the disk corona will propagate toward the disk. It will be absorbed by dust grains and re-radiated in the infrared. The local infrared spectrum will be broader than that of a single blackbody producing an equivalent loss, however, as the dust temperature will be a function of vertical distance from the corona.

The need to heat the corona points to an accretion process that is driven by magnetohydrodynamic turbulence. The extension of the disk corona into a disk wind indicates that angular momentum transport via a magnetohydrodynamic wind is also taking place. It will be interesting to ascertain if both processes are integral to the accretion of matter through a disk.

\section{References}

Appenzeller, I., Jankovics, I., \& Östreicher, R. 1984, A\&A, 141, 108

Balbus, S. A., \& Hawley, J. F. 1991, ApJ, 376, 214

Edwards, S., Cabrit, S., Strom, S. E., Heyer, I., Strom, K. M., \& Anderson, E. 1987, ApJ, 321, 473

Edwards, S., Hartigan, P., Ghandour, L., \& Andrulis, C. 1994, AJ, 108, 1056 (EHGA)

Ferreira, J., Pelletier, G., Appl, S.: 1997, in Low Mass Star Formation - from Infall to Outflow, poster proceedings of IAU Symp. No. 182, eds. F. Malbet \& A. Castets, Observ. de Grenoble, p. 112

Hamann, F. 1994, ApJS, 93, 485

Hartigan, P., Edwards, S., \& Ghandour, L. 1995, ApJ, 452, 736 (HEG)

Hartmann, L., Hewett, R., \& Calvet, N. 1994, ApJ, 426, 669

Hartmann, L., \& Raymond, J. 1989, ApJ, 337, 903

Hirth, G., Mundt, R., \& Solf, J. 1994, A\&A, 285, 929

Kwan, J. 1997, ApJ, submitted

Kwan, J., \& Alonso-Costa, J. L. 1988, ApJ, 330, 870

Kwan, J., \& Tademaru, E. 1988, ApJ, 332, L41

Kwan, J., \& Tademaru, E. 1995, ApJ, 454, 382 (KT)

Neuhäuser, R., Sterzik, M. F., Schmitt, J. H. M., Wichmann, R., \& Krautter, J. 1995, A\&A, 299, 391

Ouyed, R., \& Pudritz, R. E. 1994, ApJ, 423, 753

Paatz, G., Appl. S.: 1997, in Low Mass Star Formation - from Infall to Outflow, poster 
proceedings of IAU Symp. No. 182, eds. F. Malbet \& A. Castets, Observ. de Grenoble, p. 303

Safier, P. N. 1993, ApJ, 408, 148

Solf, J., \& Böhm, K. H. 1993, ApJ, 410, L31 